\title{
Chemically Specific Dynamic Characterization of Photovoltaic and Photoconductivity Effects of Surface Nanostructures
}

\author{
Okan Öner Ekiz,* Koray Mizrak, and Aykutlu Dâna* \\ UNAM Institute of Materials Science and Nanotechnology, Bilkent University, 06800 Ankara, Turkey
}

X -ray photoelectron spectroscopy (XPS) is a powerful spectroscopic technique for the characterization of surfaces with chemical specificity. ${ }^{1-5}$ The photoelectron spectra carries information about the binding states of different atomic species within tens of nanometers of the surface, as well as information on local potential variations. If the emitted electrons are not compensated by an external electron gun or directly from the substrate, local potentials can vary due to local conductivity variations that result in different amounts of accumulated charge. Overcharging by injection of excess electrons was previously used for surface characterization. ${ }^{6}$ Surface potentials can be shifted by direct application of a voltage to the substrate. Such shifts resulting from direct voltage stimulus were previously modeled for static and dynamic voltage excitations, taking into account conductivity and capacitances of surface domains. ${ }^{7-13}$ Nanostructures are increasingly finding application in photovoltaic technologies. Investigation of photovoltaic and photoconductivity effects in nanowires, nanocrystals, and nanocomposites are interesting from a fundamental scientific point of view. Conventional characterization techniques lack chemical specificity, and most of the time require precision fabrication of contacts on nanostructures using techniques such as electron beam lithography or related lithographies with nanoscale resolution, especially if single nanoparticles are involved. Surface photovoltage spectroscopy (SPV) has been traditionally applied to characterize photoinduced surface photovoltage changes upon illumination, using the Kelvin probe as a readout method for surface po-
ABSTRACT We report characterization of photovoltaic and photoconductivity effects on nanostructured surfaces through light induced changes in the X-ray photoelectron spectra (XPS). The technique combines the chemical specificity of XPS and the power of surface photovoltage spectroscopy (SPV), with the addition of the ability to characterize photoconductivity under both static and dynamic optical excitation. A theoretical model that quantitatively describes the features of the observed spectra is presented. We demonstrate the applicability of the model on a multitude of sample systems, including homo- and heterojunction solar cells, CdS nanoparticles on metallic or semiconducting substrates, and carbon nanotube films on silicon substrates.

KEYWORDS: X-ray photoelectron spectroscopy · carbon nanotubes · cadmium sulfide $\cdot$ silicon - dynamic characterization - surface photovoltage · photoconductivity $\cdot$ semiconductor nanoparticles $\cdot$ photovoltaics

tential shifts. ${ }^{14,15}$ Also, Kelvin probe microscopy has been used to observe photovoltaic effects on nanoscale structures. ${ }^{16}$ The chemically specific readout of photoinduced surface potential changes is a highly desirable analytical capability. Such chemically specific measurements are not possible with conventional Kelvin probe measurements. Previously, XPS has been used to probe the surface photovoltage of silicon surfaces..$^{17}$ Recently, Cohen ${ }^{18,19}$ et al. demonstrated that surface potential shifts related to external illumination were observable in XPS spectra of composite semiconductor surfaces. It was demonstrated that static or quasi-static shifts of XPS peaks can be related to photovoltaic and photoconductive effects. The shifts can be quantitatively studied with films on conductive substrates. ${ }^{18,19}$

In this article we demonstrate an approach that allows the study of photovoltaic and photoconductivity effects using the XPS, under static or modulated illumination. Surface potentials of domains are internally modulated owing to both photovoltaic and photoconductivity effects. We show that a circuit model can be used to
*Address correspondence to
ekiz@bilkent.edu.tr,
aykutlu@unam.bilkent.edu.tr.

Received for review October 15, 2009 and accepted March 30, 2010.

Published online April 9, 2010. $10.1021 / \mathrm{nn} 9014196$

๑ 2010 American Chemical Society 


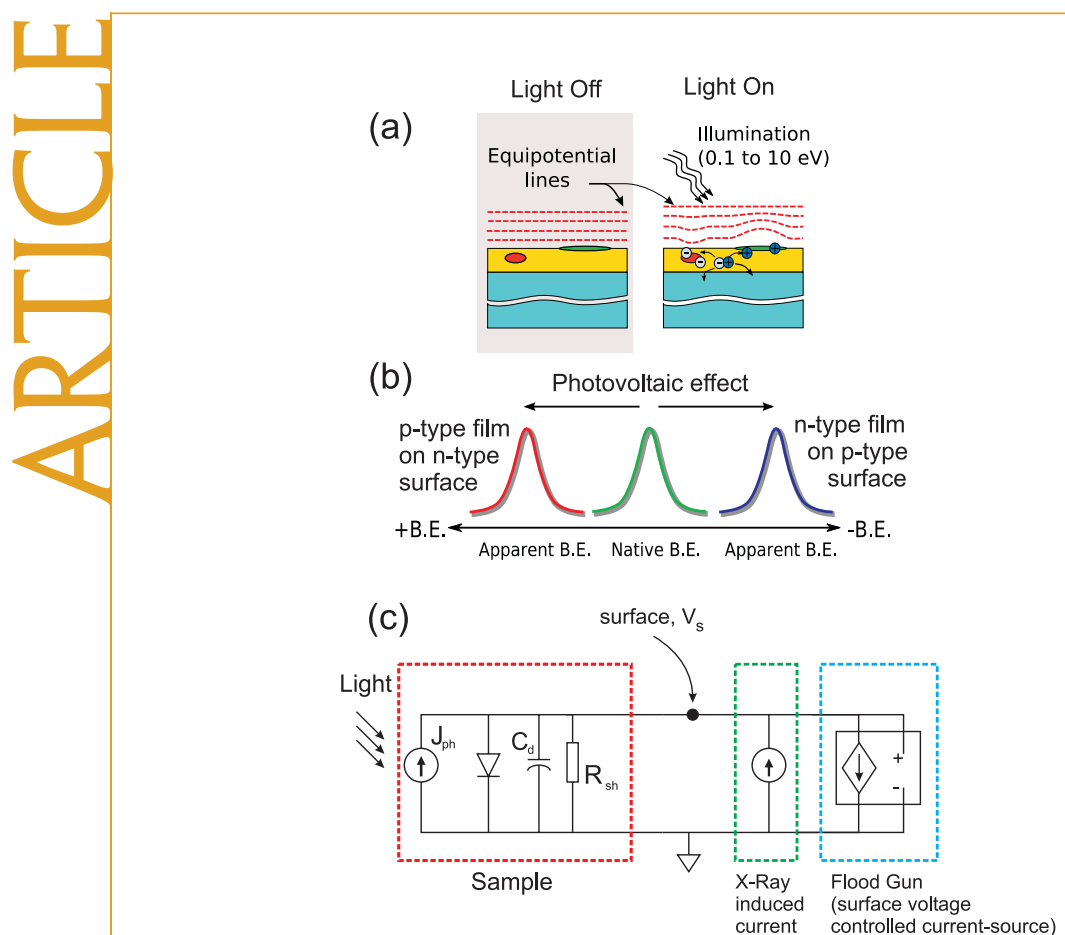

Figure 1. (a) Surface potential distribution of a composite surface containing $\mathrm{n}$ and/or $\mathrm{p}$-type domains can be perturbed upon illumination by light $(0.1-10 \mathrm{eV}$ photons) due to the photovoltaic effect. (b) Resulting potential shifts can be observed in the XPS spectra. A p-n junction is characterized by a shift of the surface potential to positive binding energies and an $n-p$ junction causes a negative shift. (c) A simplified lumped circuit model including a single junction can be used to describe static and dynamic behavior of the surface photovoltaic effect.

estimate the changes in spectra under static and dynamic illumination conditions. Light-induced surface potential differences due to photovoltaic and photoconductive effects can be identified.

The general behavior of change in surface potentials due to photovoltaic and photoconductive effects are schematically summarized in Figure 1 and Figure 2. When light is absorbed by nanoscale semiconducting domains, generated carriers diffuse and drift due to internal electric fields. Carriers may accumulate in different regions of nanostructures or escape to the substrate. Variations in surface state densities, material composition, or doping may be responsible for the creation of nanoscale junctions displaying photovoltaic effects. Photoinduced potential differences between the ground plane and surface domains can arise, as schematically shown in Figure 1a. If the domain has a p-type top surface (vacuum interface), the binding energies on the surface tend to shift toward positive energies. If the domain has an n-type top surface, the shift is toward negative binding energies. X-ray or external electron-injection related charging results in local variations of surface potentials. ${ }^{6}$ If the surface has finite conductivity, X-rays cause a positive charge build-up and the surface potential shifts toward positive energies (Figure 2b). In the presence of an electron flood-gun, the surface charge is compensated by externally in- (a)

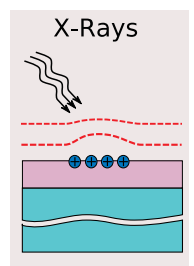

Light off

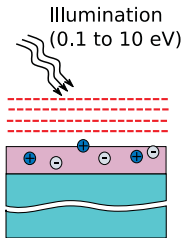

Light on

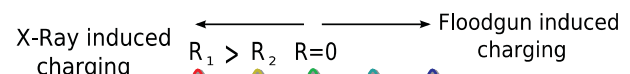

(b)

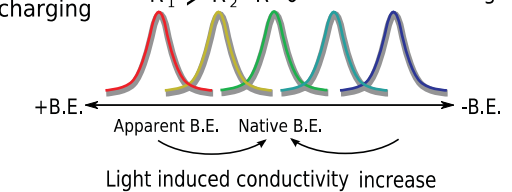

(c)

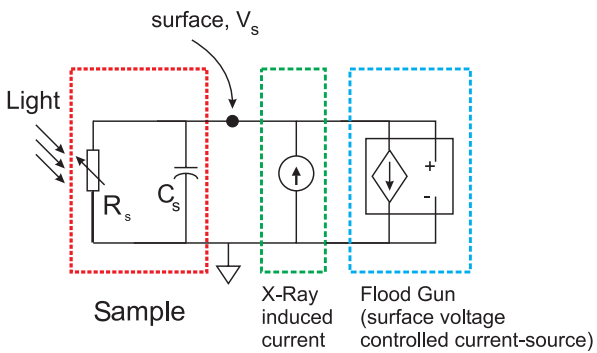

Figure 2. (a) Surface potentials can shift due to X-ray or flood-gun induced charging. (b) Excess positive charge due to X-ray illumination causes a positive shift, and excess negative charge due to electron injection by the flood-gun causes a negative shift. The shifts are proportional to the resistance between the surface and ground. Upon illumination, resistance is reduced and peaks return to the uncharged (native) binding energy. (c) A simplified lumped circuit model can be used to describe the time dependent surface potential due to static or dynamic photoconductivity.

jected electrons. If more electrons are injected than lost by photoelectron emission, the surface may be negatively charged and the spectra displays a shift toward negative binding energies. If the conductivity of the surface layer is increased by application of an external light source (photoconductive effect), the charge build-up is decreased due to compensation by available carriers. Photoconductivity causes the peaks to return closer to the native binding energy for both positively and negatively charged surfaces (Figure $2 b$ ).

In an arbitrarily complex composite film, different regions of the film may display both photoconductive and photovoltaic effects. The mechanisms shown in Figure 1 and Figure 2 may be acting in series or parallel combinations. Simple lumped circuit models as shown in Figure 1c and Figure 2c can be used to study the dynamic behavior due to photovoltaic and photoconductive effects. Simplified models include a single photovoltaic junction or a light-controlled resistance. The circuits are driven by the photocurrents generated in the junction as well as by external currents due to $X$-ray illumination and electron injection by the floodgun. The models for photovoltaic and photoconductive cases can be combined in series or parallel cascades, if both effects are simultaneously present. A feature common to all cases is the external current source. The ex- 
ternal surface current $I_{\mathrm{s}}$ can be expressed as the sum of the escaping photoelectron current $I_{x}$ (a constant, proportional to the $X$-ray photon flux intensity) and the flood-gun current $I_{\mathrm{FG}}\left(V_{\mathrm{s}}, V_{\mathrm{FG}}\right)$. The flood-gun current can be approximated by $I_{\mathrm{FG}}\left(V_{\mathrm{s}}, V_{\mathrm{FG}}\right) \cong-I_{\mathrm{FGO}}[1+\tanh$ $\left.\left(\alpha\left(V_{\mathrm{s}}-V_{\mathrm{G} 0}+V_{\mathrm{G}}\right)\right)\right] / 2$, where $V_{\mathrm{s}}$ is the surface voltage, $V_{\mathrm{FG}}$ is the flood-gun bias, $V_{\mathrm{GO}}$ is a constant offset, $l_{\mathrm{FGO}}$ is the maximum electron current achievable from the flood gun and $\alpha$ is an experimental parameter. This general form of the $I_{\mathrm{FG}}\left(V_{\mathrm{s}}, V_{\mathrm{FG}}\right)$ is chosen so that the basic physical mechanism of electron charging of surfaces are included in the model with few adjustable parameters. Electron injection by the flood-gun is dependent on the surface potential, and there are few different characteristic regimes. If the surface potential becomes negative, the flood-gun electrons are repelled from the surface causing a decrease of current. If the surface potential becomes positive, electron capture rate increases. There is a range of positive surface potentials where the electron current is linearly proportional to the surface potential. Owing to the finite availability of electrons, above a certain positive surface voltage, electron current does not further increase and reaches a saturation value $I_{\mathrm{FG} 0}$.

\section{RESULTS AND DISCUSSION}

We investigate the validity of the model by considering samples which demonstrate dominantly photovoltaic or photoconductive effects, as well as the simultaneous presence of both. It is known that light-induced carrier generation and diffusion may disturb the surface pinning and can cause band bending in semiconductors. Therefore, bare semiconductor surfaces may show some degree of surface photovoltaic (SPV) effect. The magnitude and sign of the surface photovoltaic effect depend on many parameters, such as the distribution of surface density of states, the bulk Fermi level position, and mobilities. Such SPV effects can be observed using the XPS, as is demonstrated in Figure 3. Since silicon substrates are used in the experiments involving nanoparticles, we first characterize the SPV of the substrates. When an n-type silicon surface (nominal resistivity $1-10 \mathrm{Ohm}-\mathrm{cm}$ ) is illuminated with white light, there is no identifiable shift (Figure 3a). However, when a p-type silicon surface (resistivity $1-10 \mathrm{Ohm}-\mathrm{cm}$ ) is illuminated with white light, a surface potential shift of $-0.13 \mathrm{eV}$ is observed (Figure $3 \mathrm{~b}$ ). Such shifts have been previously characterized as a function of doping density and type. It is known that p-type silicon produces a greater shift for a given doping level. ${ }^{14} \mathrm{~A}$ greater doping level is known to reduce the SPV. Our observation is in agreement with the previous studies, if it is assumed that the actual doping level of the n-type substrate is higher than p-type. To unambiguously demonstrate photovoltaic effects, ideal $p-n$ and $n-p$ junctions are studied. Two silicon solar cells were studied. The photovoltaic effect can be observed as shown
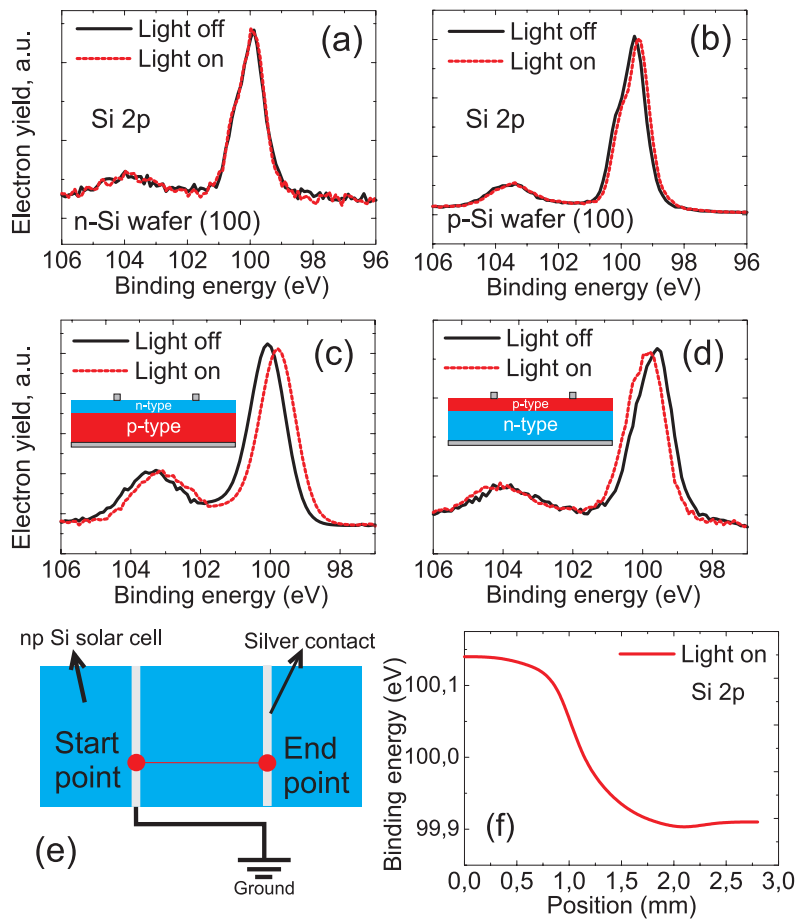

Figure 3. Surface potential of a bare silicon surface is not significantly affected by illumination for an (a) $n$-type substrate, but (b) a shift of $-0.13 \mathrm{eV}$ is observed for a $p$-type substrate. When fabricated (c) $n-p$ and (d) $p-n$ junctions are illuminated; apparent binding energy shifts by $V_{\mathrm{OC}}$ are observed. Note that the signs of the shifts are as expected, negative for the $n-p$ junction and positive for the $p-n$ junction. (e) Light induced surface potentials can also be mapped spatially by measuring at different locations on the sample.

in Figure $3 c$ and Figure $3 d$ on both $n-p$ and $p-n$ junctions. The Si $2 p$ peak in the XPS spectra of an $n-p$ junction (top side $n$, bulk of the substrate $p$-type) from a commercial monocrystalline solar cell is shown to shift toward negative binding energies (Figure 3c). The shift direction is reversed for the $\mathrm{p}-\mathrm{n}$ junction (top side p-type, bulk of the substrate n-type). The magnitude of the shift can be estimated using the model in Figure $1 \mathrm{c}$. The shunting resistance $R_{S H}$ in the model may be an internal resistance, or may be due to grounding of the top surface by an external resistance. In our XPS system, the samples are mounted using insulating clamps or bronze clamps, which act as resistive grounding paths. The effect of the extra ground path is observed in the surface potential for an $n-p$ silicon junction (Figure $3 e$ and Figure $3 f$ ). As the spectra is collected close to a grounded metal contact, surface potential shifts are decreased. As we move further away from the grounded contact, the surface potential shift increases to negative values (Figure 3f). The deviation of the surface potential from linear can be understood using a distributed circuit model. We can divide the photovoltaic cell into smaller cells connected to each other by small series resistances. Because of the nonlinear IV characteristics of individual cells, we observe an abrupt shift of the surface potential. The location of the abrupt 


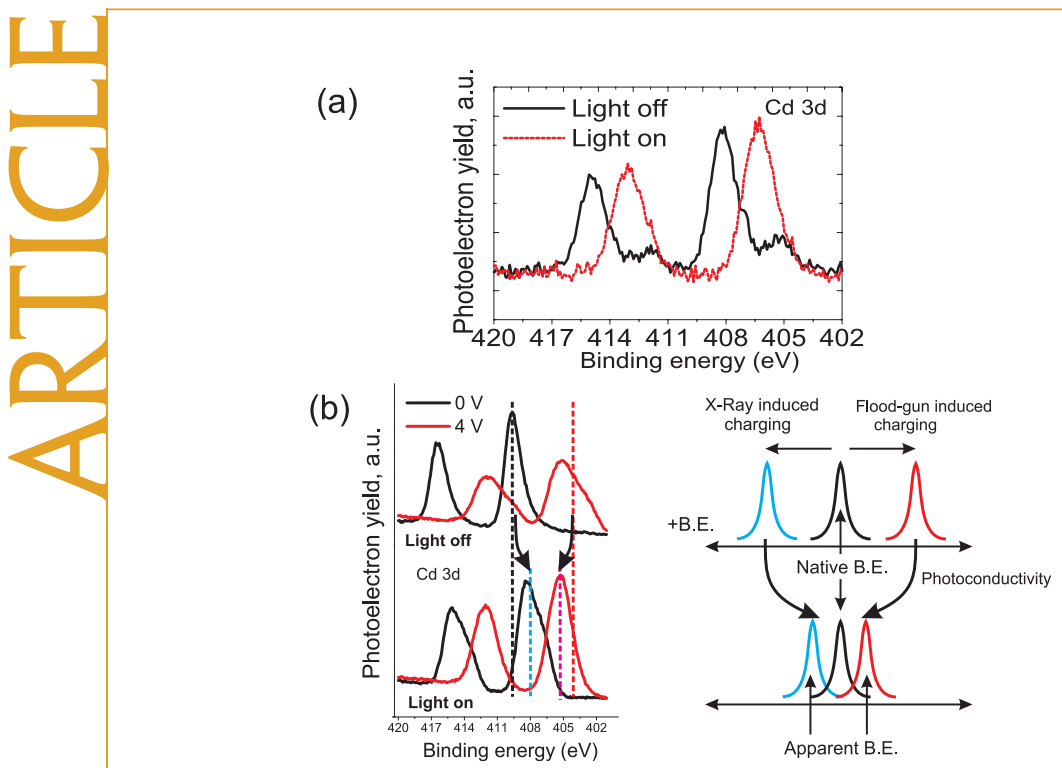

Figure 4. (a) Photoresponse of CdS nanoparticles on a grounded AuPd surface; (b) photoresponse of CdS nanoparticles on n-type silicon (experimental data, left), where X-ray charging is dominant $\left(V_{\mathrm{FG}}=0\right)$ and electron charging is dominant $\left(V_{\mathrm{FG}}=4 \mathrm{~V}\right)$ in the dark. In both cases, illumination causes reduction of charging. The peaks tend to shift toward the native binding energy (schematic description, right).

change depends on the ratio of cell currents and circuit resistances. The XPS data is a result of the convolution of the spot-size of the spectrometer and spatial variations of the surface potential. This causes smoothing of the voltage profile.

To demonstrate photoconductive effects, CdS nanoparticles were studied on a variety of substrates. XPS spectra of $\mathrm{CdS}$ particle film on a grounded $\mathrm{Au} / \mathrm{Pd}$ surface were recorded under dark and illuminated conditions as shown in Figure 4a. Here the flood-gun is turned off, and all charging is due to X-ray induced charging. In the spectra, two distinct peaks are identifiable in the $\mathrm{Cd} 2 \mathrm{~d}$ spectrum. In the dark, X-ray charging is seen to cause a shift of one of the peaks toward positive binding energies ( $408.15 \mathrm{eV}$ for $\mathrm{Cd}$ ). As the sample is flooded with white light from a superbright LED, this peak shifts toward the native binding energy of $\mathrm{Cd}$ in $\mathrm{CdS}$. The smaller peak in the spectrum, originally at $405.4 \mathrm{eV}$ shifts only $0.1 \mathrm{eV}$ to 405.33 , which is very close to the original position of cadmium peak for $\mathrm{CdS} .^{20} \mathrm{We}$ attribute this peak to the particles that are directly in contact with the surface (regions with monolayer or submonolayer coverage).

The charging effect due to the flood-gun electrons is also shown in Figure 4b. XPS spectra of a CdS nanoparticle film on a n-doped silicon substrate is recorded under dark and illuminated conditions. The flood-gun voltage $V_{G}$ was set to 0 and $4 \mathrm{~V}$. The convention for the sign of the flood-gun voltage is equipment dependent. In our case a positive $V_{\mathrm{G}}$ causes increased electron charging. Under dark conditions and $V_{G}=0 \mathrm{~V}$, a positive shift is observed. The positive shift is a result of $\mathrm{X}$-ray related charging. For $V_{G}=4 \mathrm{~V}$, electron charging becomes dominant, and a negative shift of the $\mathrm{Cd}$ peak is observed. As the sample is illuminated, both positive and negative shifts decrease. The peaks return closer to the native binding energy. This is attributed to the increase in the conductivity of the CdS film.

A more general case is the simultaneous presence of both photoconductivity and photovoltaic effects. Band alignment and photovoltaic effects of $\mathrm{CdS} / \mathrm{p}$-Si heterojunctions has been previously studied. ${ }^{21,22}$ In general photoconductive effect results in a decrease of the charging related shifts and photovoltaic effects cause an increase in the magnitude of shifts. However, with multiple unknowns about a specific sample, it is not possible to separate the two effects without the addition of a swept parameter. The flood-gun potential can be used for this purpose. The surface potential shifts as the flood-gun bias is changed from 0 to $4 \mathrm{~V}$, inducing controlled hole or electron charging. Illumination reduces the resistance, and magnitudes of positive and negative shifts are both reduced. When the peak positions are plotted versus flood-gun potential, if the photovoltaic effect is not present, the curves for dark and illuminated conditions show a crossingover behavior. This is because there exists a flood-gun potential $V_{G, 0}$ for which the $\mathrm{X}$-ray induced current is canceled by electron injection. At this flood-gun voltage, charging is zero regardless of the sample resistance $R_{5}$, causing the dark/light curves to cross. If a photovoltaic effect is present, the curves may not cross owing to the shift caused by the photovoltage. The crossing-over or noncrossing over behavior depends on the relative strengths of the photovoltaic and photoconductive effects. The sign of the photovoltage is also a factor in the determination of the crossing/anticrossing behavior. We demonstrate these effects using samples that show both photoconductivity and photovoltaic effects are present. We measure the XPS spectra of films of $\mathrm{CdS}$ nanoparticles on $\mathrm{n}$ - and $\mathrm{p}$-type silicon as a function of $V_{G}$ (Figure 5). CdS forms an $n-p$ junction on p-type silicon and displays both photovoltaic and photoconductive effects. CdS films on an n-type silicon substrate show photoconductivity dominantly. For n-type silicon, a crossing of the peak energies is observed under dark and illuminated conditions. The experimental data in Figure 5a can be qualitatively reproduced assuming the presence of a photoconductive effect Figure 5b. This observation suggests the absence of a strong photovoltaic effect between the $n$-type CdS film and n-type Si. For CdS on p-type silicon, the photoconductivity of the nanoparticle film is accompanied by the photovoltaic effect between the $\mathrm{CdS} / \mathrm{p}$-type silicon.

The wavelength dependence of the effects can be seen in Figure $5 c$. The $\mathrm{CdS}$ film on a p-type silicon substrate is illuminated with 532 (2 $\mathrm{mW}$ nominal power) and $650 \mathrm{~nm}$ (1 mW nominal power) lasers. CdS nano- 

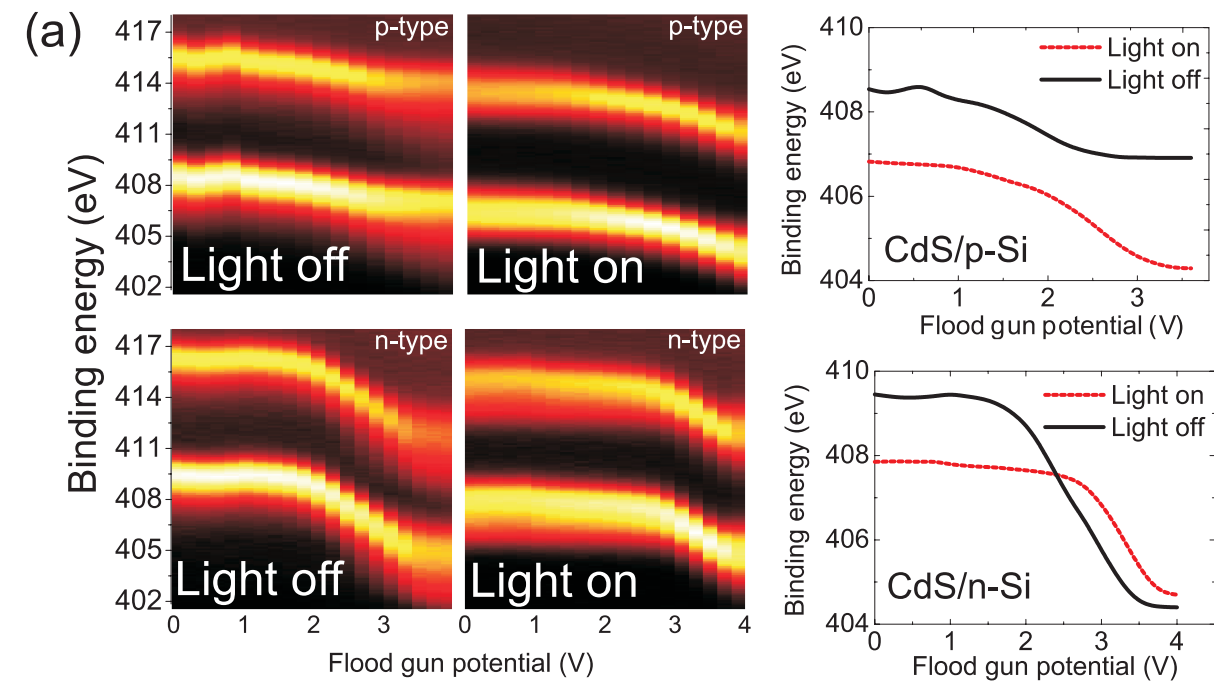

(b)
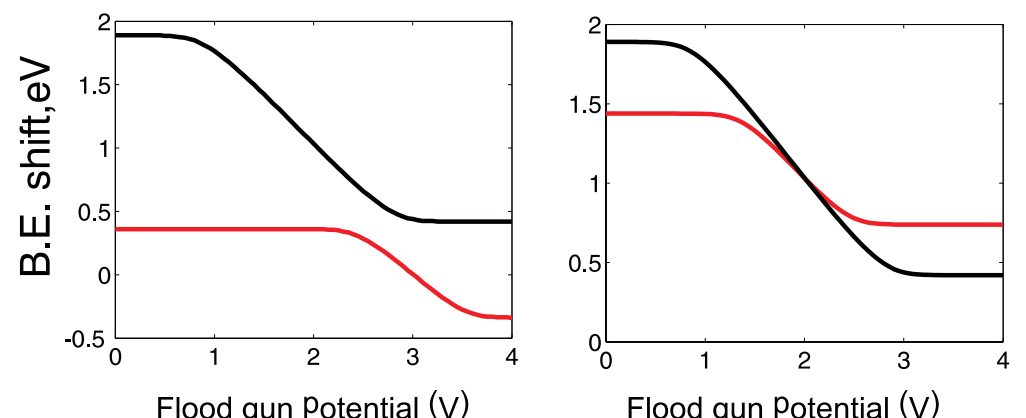

(c)

$532 \mathrm{~nm}$
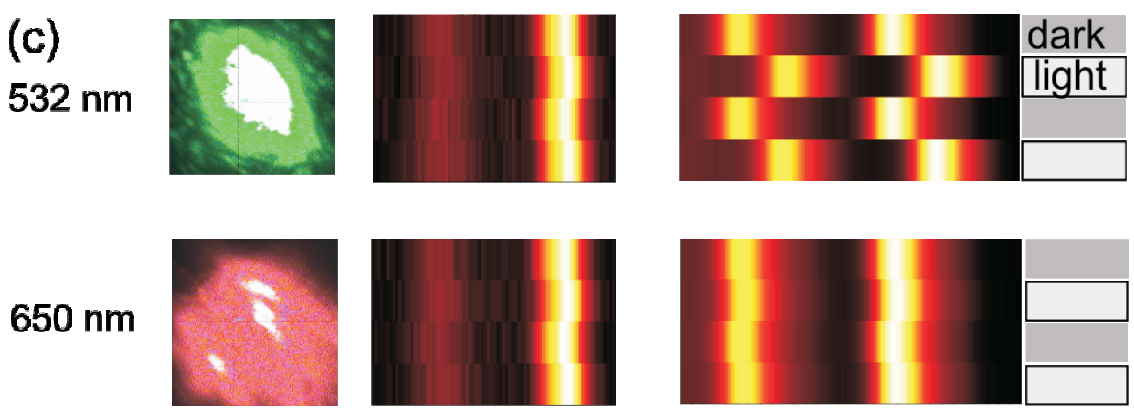

Silicon

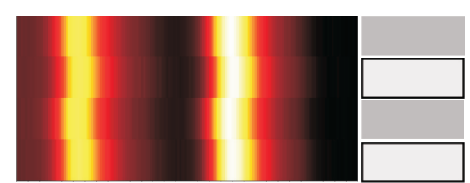

Cadmium

Figure 5. (a) Light-induced changes in the XPS spectra of Cd from CdS nanoparticles on $n$ - and p-type silicon substrates as a function of the flood-gun potential. Peak positions are plotted for dark and illuminated cases showing significantly different behavior. (b) Theoretical model can be used to predict a similar behavior for the $\mathrm{n}$-type CdS on $\mathrm{n}$ - and $\mathrm{p}$-type silicon. The change from crossing to noncrossing curves are attributed to the presence of a photovoltaic effect for the $n-C d S / p-S i$ case. The anticrossing behavior is not universal but is dependent on experimental and sample related parameters such as the total induced photovoltage at the junction, floodgun and X-ray induced surface currents, and strength of the photoconductivity effect. (c) Photoconductive and photovoltaic effects are also shown to be wavelength dependent: When illuminated by $532 \mathrm{~nm}$ light (above the CdS bandgap), both CdS and silicon display light-induced effects, silicon showing only surface photovoltaic effect and CdS displaying both photovoltaic and photoconductive effects. When illuminated by a $650 \mathrm{~nm}$ laser, the sample absorbs light only in the p-type silicon substrate producing shifts equal to the silicon surface photovoltage shown in Figure $3 \mathrm{~b}$. In this case, the apparent CdS binding energy follows the potential of the silicon substrate it rests upon.

particles have a bandgap above $2.42 \mathrm{eV}^{23}$ therefore no absorption is expected for $650 \mathrm{~nm}$ illumination in the CdS layer. Here, the flood-gun is turned off and all charging is due to X-ray exposure. The CdS layer remains charged under $650 \mathrm{~nm}$ illumination. However, p-type silicon shows a shift of $-0.13 \mathrm{eV}$ (as was previously observed on a bare p-type sample, shown in Figure $3 b)$. Since the CdS film is referenced to the silicon surface, the apparent binding energy of $\mathrm{Cd} 3 \mathrm{~d}$ is also shifted by $-0.13 \mathrm{eV}$. When the sample is illuminated using $532 \mathrm{~nm}$ laser, both CdS and Si display photoactivity, Cd3d shifting as much as $-2.5 \mathrm{eV}$. The photoconductivity related shift of the $\mathrm{Cd} 3 \mathrm{~d}$ line on an n-type substrate is about $-2.1 \mathrm{eV}$, with no significant shift of the Si peak. We therefore conclude that the $\mathrm{CdS} / \mathrm{p}-\mathrm{Si}$ junction displays a photovoltage of about $-0.27 \mathrm{eV}$. For 


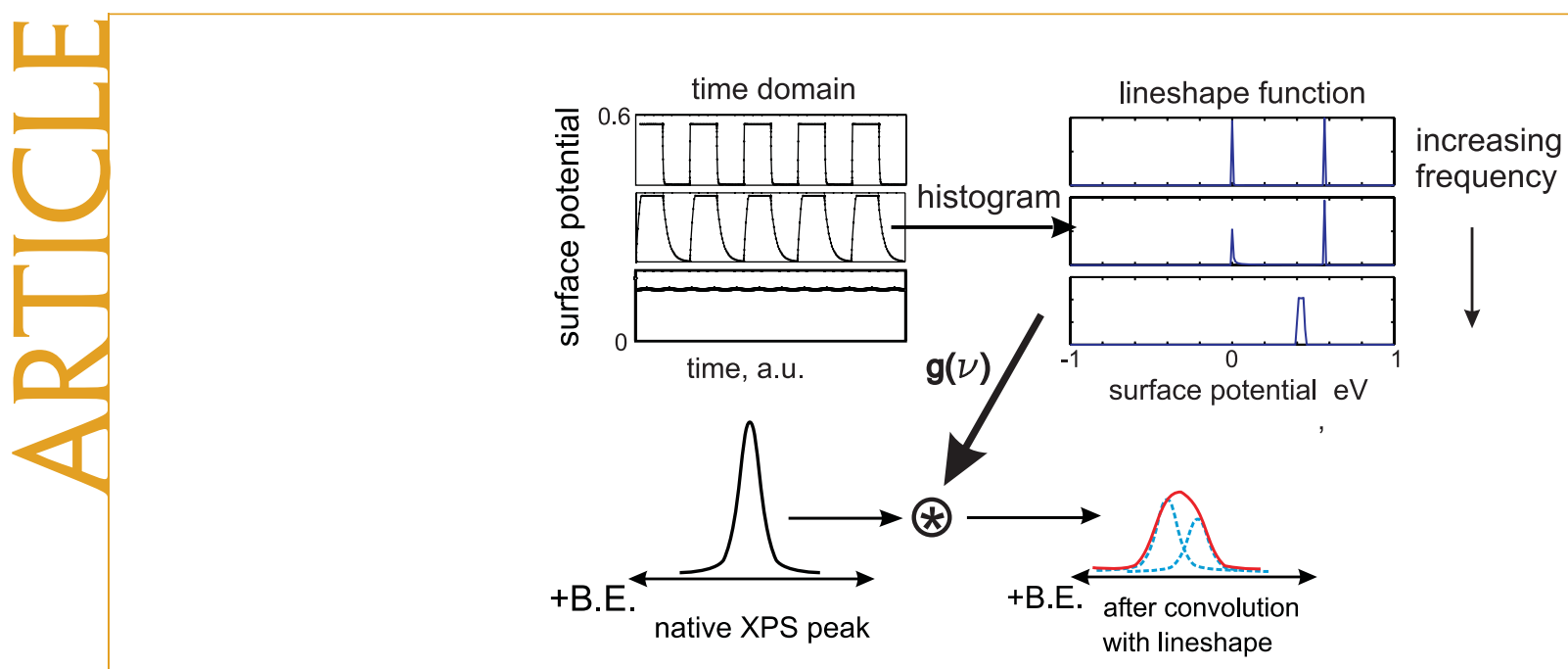

Figure 6. Surface voltages under modulated illumination are calculated using the simplified circuit model. Time varying surface potentials are used to calculate line shape function $g(v)$ using a histogram. The line shape function is then convoluted with the original unmodulated XPS data to predict spectra under modulated illumination.

CdS/p-type silicon, there are two photovoltaic junctions (CdS/p-Si and p-Si surface) and a single photoconductive region (CdS), all acting in series. Wavelength dependent measurement allows us to distinguish shifts due to individual junctions.

Previously dynamic characterization of surface voltage distributions were carried out using externally

(a)
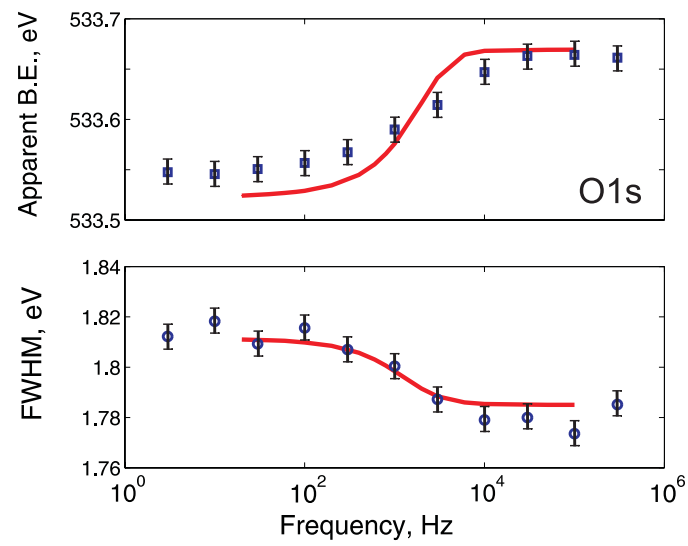

(b)

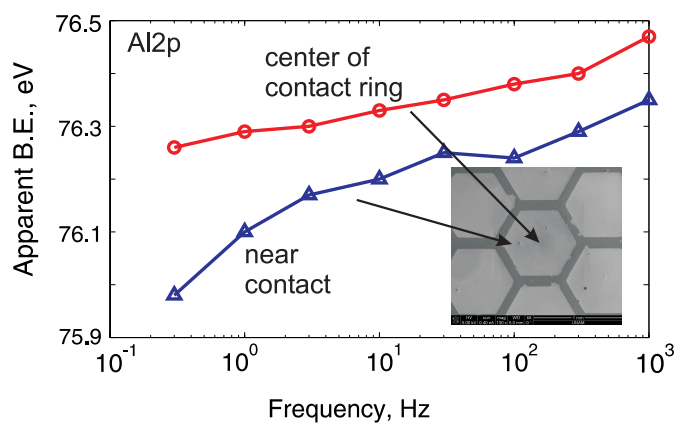

Figure 7. (a) Apparent binding energy and full-width-at-halfmaximum of the oxygen peak on a $p-n$ ALIC cell as a function of modulation frequency is shown. Theoretical model assumes $V_{\mathrm{OC}}=0.53 \mathrm{~V}$ and $R_{\mathrm{SH}}=850 \Omega$ extracted from the $I-V$ measurements on the same cell. The capacitance value $(C=200 \mathrm{nF})$ is obtained by fitting. (b) The frequency dependence of apparent binding energies of residual aluminum, collected at points close to and away from the contact, is shown. Slightly different frequency responses are observed. modulated sample bias. A simplified RC model of a composite surface was shown to quantitatively predict XPS spectra. The convolution approach used for simulation of XPS spectra under dynamic excitation ${ }^{24}$ can be extended to include the photoeffects using the model shown in 1c. A p-n junction (ALIC cell) is placed inside the XPS chamber and subjected to static and modulated illumination by an external laser $(650 \mathrm{~nm}$, multimode diode laser, $0.5 \mathrm{~mW}$ ) with modulation frequencies ranging from $0.1 \mathrm{~Hz}$ to $1 \mathrm{MHz}$, while the photoelectron spectra are collected. The surface potentials of various domains on the device are modulated according to their local electronic properties. The electronic properties determine the equivalent lumped circuit elements such as capacitances, shunt, and series resistances.

We demonstrate the applicability of the technique on a $\mathrm{p}-\mathrm{n}$ junction known to display the photovoltaic effect dominantly. We use the model in Figure 1 in a circuit simulator to calculate the dynamic response of the lumped circuit. The time-dependent surface potentials are calculated with the simulator, then the line shape functions $g(v)$ are calculated using a histogram of the time-series of the surface voltage. The lineshapes are then used to estimate the peak position shifts and broadenings as described elsewhere. ${ }^{25}$ It is seen that, if the frequency of modulation is slow compared to the characteristic cutoff frequency of the surface response, the line shape function resembles a double delta function separated by $V_{\text {OC }}$ (Figure 6). The resulting convolved peak shape has a greater width and reduced peak intensity. The peak position is shifted to about $V_{\mathrm{OC}} / 2$. As the modulation frequency is increased above the cutoff frequency of the junction, the junction acts like a low-pass filter. In this case, the line shape becomes like a single delta function. The width of the observed peak becomes narrower, closer to the native width of the peak. This is accompanied by a slight shift of the peak position toward $V_{\mathrm{OC}}$. Simulation results for 
the ALIC cell, using parameters extracted from dark and illuminated $I-V$ curves are shown in Figure 7 . The model predicts the correct form of frequency dependence. It is seen that when model parameters obtained by conventional device characterization methods are used, theoretical calculation of the peak shifts and broadening agree with the XPS measurements. This observation suggests that model parameters of solar cells can be extracted through numerical analysis and using XPS measurements only. In principle, correlations of optoelectronic properties can be established between different peaks automatically. Similar correlations of electronic properties were extracted using modulated external bias. ${ }^{24,26}$ Location dependent dynamic characterization is also possible. Changes in the SPV frequency response can be observed if the response is measured close to or away from the contacts (Figure 7b)

The photoconductivity can be studied dynamically as well as through photovoltaic effects. We use pulsed illumination on a CdS nanoparticle film on an n-type substrate. Light pulses of duration $\tau_{\text {pulse }}=5 \mathrm{~ms}$ with a repetition rate $f_{\text {rep }}$ is incident on the sample. A superbright white LED is used as the light source. The flood gun is turned off and only X-ray gun charging is present. During the light pulse, photoconductivity reduces the resistance of the film $\left(R=R_{\text {on }} \approx 0\right)$. In this period, the surface may be thought as shorted to ground. During the dark period, the surface behaves like an an RC circuit $\left(R=R_{\text {off }} \approx 30 \mathrm{M} \Omega, C \approx 0.2 \mathrm{nF}\right.$ ), charged by the X-ray current $\left(I_{\mathrm{x}} \simeq 100 \mathrm{nA}\right)$. The circuit element values are obtained by fitting. If the pulse repetition rate is increased, the film spends more time in the short state. Therefore, at low frequencies the film is mostly charged and at high frequencies the film is mostly shorted. The gradual transition between fully off and fully on states can be observed in the XPS data (Figure 8a). When the pulse frequency is close to the effective RC time constant of the film, maximal peak broadening occurs. This feature, theoretically estimated by the model, can be observed in the experimental results shown in Figure 8b.

We apply the technique to the characterization of light induced changes in the XPS spectra of single and multiwalled carbon nanotubes (SWCNT and MWCNT) on metal and semiconductor substrates. Electronic structure and properties of SWCNTs and MWCNTs have been extensively studied both theoretically and experimentally. ${ }^{27-33}$ Also CNTs have found application in photovoltaic devices both as contacts and active layers. ${ }^{34-40}$ For the sake of simplicity, the experiments were performed without activating the flood-gun. In this case, all charging must be due to $\mathrm{X}$-ray related charging. It is observed that the carbon peak from a thin film of MWCNTs on a n-type silicon surface demonstrates a shift of $+0.3 \mathrm{eV}$ upon illumination (Figure 9b). No significant shifts are observed on p-type silicon or on AuPd-coated substrates. A similar shift is observed with a SWCNT film on n-type silicon substrates. When
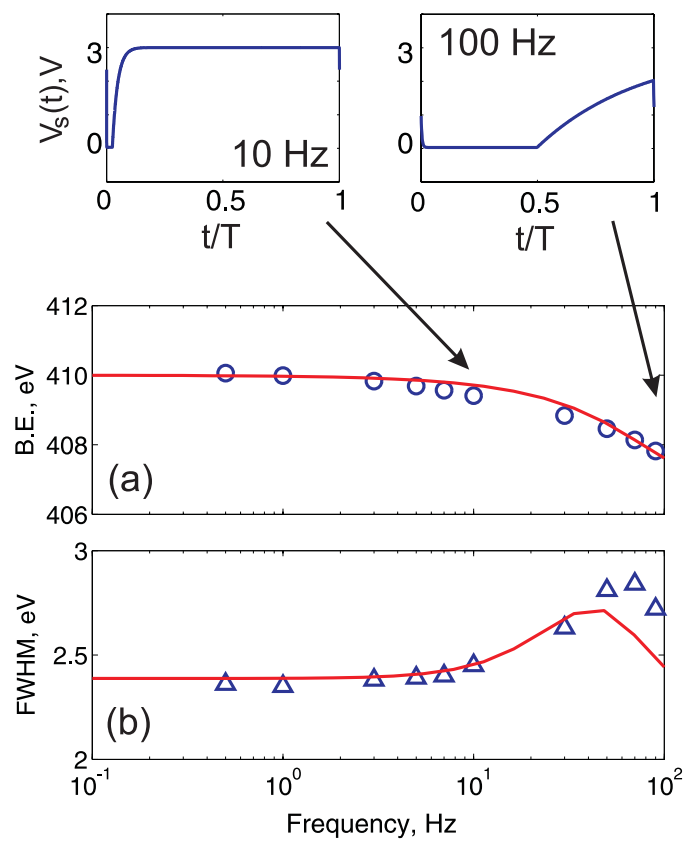

Figure 8. The effect of pulsed illumination on the $\mathrm{Cd} 3 \mathrm{~d}$ peak for a CdS nanoparticle film on n-type silicon. Because of modulation of CdS film conductivity by a $5 \mathrm{~ms}$ long light pulse of varying repetition frequency, the charging is intermittently reduced. The flood-gun is turned off and X-ray related positive charging is present only. (a) The peak position shifts as a function of repetition frequency $f_{\text {rep }}$ (b) Broadening of the peak is observed when pulse-to-pulse separation time is near the effective $R C$ time constant of the film. Experimental observations can be understood using the model given in Figure 2. $R$ and $C$ values, as well as the $X$-ray induced current $l_{\mathrm{x}}$ are fitting parameters. Satellite graphs show theoretically calculated transient surface potentials for slow and fast pulse repetition rates.

the native oxide layer (typically few $\mathrm{nm}$ thick) on the substrate is cleaned using HF prior to casting of the CNT film, it is observed that the light induced shift is decreased (Figure 9e). Since only X-ray charging is possible without the flood-gun, in this case the photoconductive effect may only produce a negative shift, in favor of reducing charging. As the observed lightinduced shift is positive, it is due to the photovoltaic effect, the CNT film acting effectively as a p-type domain on an n-type substrate. This conclusion is also supported by observation of the effect only on n-type substrates. To understand the origin of the observed shift, we model the electrostatic problem at the CNT/oxide/ silicon interface under illumination using rate equations, taking into account the generation-recombination and electron/hole tunneling rates as shown in Figure 9a. We assume carrier generation in the CNT film only. In CNTs, the electron and hole properties are highly symmetric, ${ }^{28,41}$ and we may assume a density of states for the CNT ensemble symmetric around the CNT affinity of $4.5 \mathrm{eV} . .^{35,42,43}$ We assume that the relaxation inside the CNT film is rapid, and the CNT film is in equilibrium, with separate quasi Fermi levels for electrons and holes. We also assume that the escape rates of carriers to the substrate are 

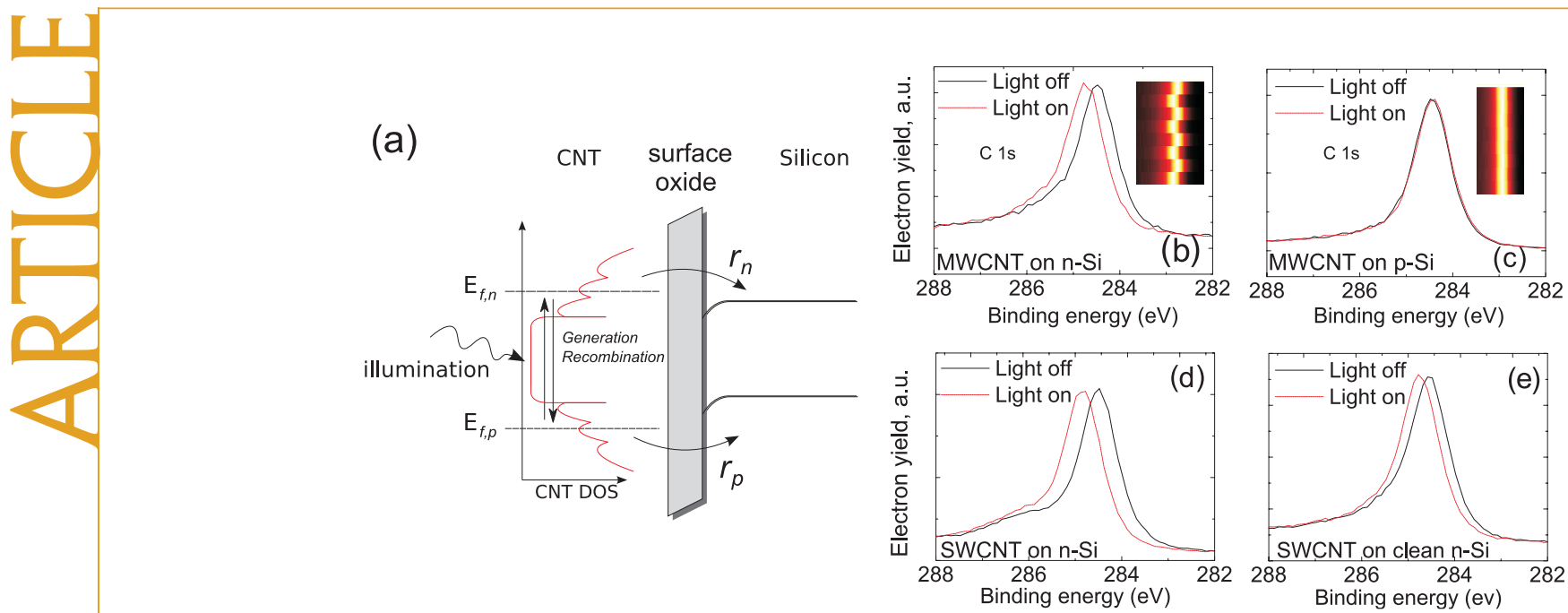

Figure 9. (a) Schematic description of the band alignment of carbon nanotube film on silicon under external illumination; (b) light-induced shifts can be observed in the carbon spectrum with a multiwall carbon nanotube film on an $n$-type substrate; (c) no significant shift is observed on a p-type silicon substrate. Insets show repeatability of shifts under on-off illumination; (d) slightly larger shifts are observed for single-wall nanotubes on an $n$-type silicon substrate with native oxide; (e) ILight induced shifts are reduced after removal of the native oxide with HF.

dominated by direct tunneling rates. Under these simplifying assumptions, the net charge on the CNT film and resulting surface potential is determined by an imbalance between the electron and hole tunneling rates $r_{\mathrm{n}}$ and $r_{\mathrm{p}}{ }^{44,45}$ Under illumination, carrier generation and recombination rates, $G$ and $R$ are related to escape rates and carrier concentration change rates by $\partial \Delta n / \partial t=G$ $-R-\Delta n r_{\mathrm{n}}\left(F_{\mathrm{ox}}\right)$ and $\partial \Delta p / \partial t=G-R-\Delta p r_{\mathrm{p}}\left(F_{\mathrm{ox}}\right)$ where $F_{\text {ox }}$ is the barrier (oxide) field. At a given time, the electron and hole concentrations in the CNT film are $\Delta n$ and $\Delta p$, giving a net charge concentration of $\Delta q=\Delta p-$ $\Delta n$. The barrier field is related to charge on the CNT film by $F_{\mathrm{ox}} \simeq \Delta q / 2 \varepsilon_{\mathrm{ox}}$. Under steady state conditions, $\partial \Delta n / \partial t$ $=\partial \Delta p / \partial t=0$ and $\Delta n r_{\mathrm{n}}\left(F_{\mathrm{ox}}\right)=\Delta p r_{\mathrm{p}}\left(F_{\mathrm{ox}}\right)$. The surface potential shift $\Delta V_{\mathrm{s}} \simeq F_{\mathrm{ox}} t_{\mathrm{ox}}$, where $t_{\mathrm{ox}}$ is the oxide (barrier) thickness. The net charge can be rewritten as $\Delta q=\Delta p$ $-\Delta n=\Delta n\left(r_{\mathrm{n}}\left(F_{\mathrm{ox}}\right) / r_{\mathrm{p}}\left(F_{\mathrm{ox}}\right)-1\right)$. The tunneling rates $r_{\mathrm{n}}$ and $r_{\mathrm{p}}$ are assumed to be determined by direct tunneling. For small barrier fields $\left(F_{\mathrm{ox}} \approx 0\right), r_{i} \propto \exp \left(-3 B_{\mathrm{i}} \vee V_{\mathrm{B},} t_{\mathrm{ox}} /\right.$ 2), where $B_{i}=4\left(2 m_{\mathrm{ox},} q\right)^{1 / 2} / 3 \hbar ; V_{B, i}$ is the barrier height for tunneling and $m_{\mathrm{ox}, i}$ is the tunneling effective mass. ${ }^{46-48}$ Hence, tunnel rates are exponentially dependent on the electron and hole barriers $\left(V_{B, \mathrm{e}} \simeq 4.5\right.$ and $V_{B, p} \simeq 4.66 \mathrm{eV}$ ) and barrier thickness $t_{\mathrm{ox}}$, electron tunneling being typically faster. Hence, a positive net charge accumulates on the CNT film and a surface potential shift $\Delta V_{s}$ proportional to the oxide thickness is expected. As the surface potential shift increases, changing barrier field tends to readjust the electron and hole escape rates and an equilibrium value proportional to the oxide thickness is achieved. The estimations of this model agree with the observations shown in Figure $9 d$ and Figure 9e, where a decrease of the surface photovoltage is seen for reduced barrier thickness. The observed photovoltage polarity is in parallel with previous demonstrations of solar-cells made up of carbon-nanotube films on n-type silicon. ${ }^{39}$

\section{CONCLUSION}

We present an extended model of surface potentials in an XPS experiment in the presence of photovoltaic and photoconductive effects. The approach is shown to distinguish between surface voltage shifts due to photovoltaic or photoconductive effects. The model also can be used to predict dynamical behavior of surface voltages under modulated illumination. Wavelength dependent absorption and chemically specific response is observed in composite surfaces. The origins of the shifts can be understood by referring to the predictions of the presented model. The method can be used in combination with standard XPS procedures, such as depth profiling and angle resolved XPS, to provide greater insight into the origins of optoelectronic effects. The demonstrations also underline the importance of illumination in an XPS experiment.

\section{EXPERIMENTAL SECTION}

The surface photovoltaic effects were observed on shallow $p-n$ and $n-p$ junctions. The $n-p$ junction was a commercial silicon photodiode produced by $\mathrm{POCl}_{3}$ based diffusion. The $\mathrm{p}-\mathrm{n}$ junction was made in-house by metal induced crystallization. The device studied in the experiments were fabricated by depositing a nominally $400 \mathrm{~nm}$ thick amorphous silicon (a-Si) layer onto a hydrofluoric acid (HF) cleaned n-type silicon wafer (1-10 $\mathrm{Ohm} \mathrm{cm}$ nominal resistance) using plasma enhanced chemical vapor deposition (using $\mathrm{SiH}_{4}$ as the silicon source, at $250{ }^{\circ} \mathrm{C}$ ). The a-Si layer was then partially crystallized by thermal evaporation of a thin ( $50 \mathrm{~nm}$ mass thickness) Al layer and annealing in a $\mathrm{N}_{2}$ atmosphere at $500{ }^{\circ} \mathrm{C}$ for $30 \mathrm{~min}$. Partial aluminum induced crystallization (ALIC) occurs around hot spots on the surface, and unreacted aluminum was cleaned using HF. Contact pads for the $\mathrm{p}$-type (aluminum, front) and n-type (gold, back) regions were fabricated using lithography and thermal evaporation. The contacts were not further annealed. Aluminum also dopes the 
crystallized silicon causing formation of p-type domains. ${ }^{49} \mathrm{It}$ was observed that in our process, aluminum silicide/oxide inclusions are formed near the surface as observed by electron microscopy XPS and EDS analysis (data not shown). The resulting device performs as a solar cell with a relatively low fill-factor and efficiency (efficiency $=6.4 \%$ obtained by comparing to a monocrystalline reference cell of known efficiency, data not shown) with an open circuit voltage of $V_{o c}=0.55 \mathrm{~V}$ and short circuit current of $6.5 \mathrm{~mA} / \mathrm{cm}^{2}$. The series and shunt resistances are estimated to be $R_{\mathrm{s}}=52 \Omega$ and $R_{\mathrm{sh}}=850 \Omega$ for a $1.5 \mathrm{~cm}^{2}$ device. Several analytical methods were used to characterize the structure of the device, including cross-sectional scanning electron microscopy, EDS analysis, and confocal Raman microscopy (data not shown). It was observed that aluminum rich inclusions are present on the surface with sizes ranging from 50 to $400 \mathrm{~nm}$ vertically and from $50 \mathrm{~nm}$ to few micrometers in the sample plane. It was observed in the Raman spectra that a-Si is partially crystallized around the inclusions. The shunt resistance $R_{\mathrm{sh}}$ can be due to multiple effects: fast recombination inside the polycrystal layer due to native defects and due to defects introduced by aluminum, as well as nanoscale shorts due to the presence of large AlSi inclusions that run between the top of the p-type surface and the n-type substrate.

$\mathrm{CdS}$ nanoparticles were synthesized by a solvothermal process. ${ }^{50}$ Cadmium sulfide nanoparticles with average diameter of $60 \mathrm{~nm}$ were prepared by mixing cadmium acetate $\left(\mathrm{Cd}\left(\mathrm{CH}_{3} \mathrm{CO}_{2}\right)_{2}\right)$ thiourea in ethylene glycol with a molar ratio of $1 / 8$. The mixture was heated for $1 \mathrm{~h}$ at $180^{\circ} \mathrm{C}$. After cooling, the CdS nanoparticles precipitated. The particles were separated from the solvent by centrifugation. The particles were washed three times with ethanol. Substrates were prepared by simple dip coating with ethanol suspended CdS nanoparticles solution and then dried at $100{ }^{\circ} \mathrm{C}$. SEM and SAXS measurements showed that the diameter of the CdS nanoparticles is around $60 \mathrm{~nm}$. EDX measurements provide evidence that the particles are composed of $\mathrm{Cd}$ and $\mathrm{S}$ and XRD measurements showed the particles are nanocrystalline CdS.

The MWCNTs are commercially obtained and are around 50 $\mathrm{nm}$ in width and $10-15 \mu \mathrm{m}$ in length. MWCNTs were dissolved in dicholoromethane (DCM) and sonicated for $1 \mathrm{~h}$. A similar procedure was used for SWCNTs. After sonication the substrates were prepared by dip coating with SWCNTs-DCM or MWCNTs-DCM solution than dried at $100^{\circ} \mathrm{C}$.

Acknowledgment. This work was partially funded by TUBITAK 107T547, and by the State Planning Agency of the Turkish Republic Project UNAM.

\section{REFERENCES AND NOTES}

1. Siegbahn, K.; Hammond, D.; Fellner-Feldegg, H.; Barnett, E. F. Electron Spectroscopy with Monochromatized X-rays. Science 1972, 176, 245-252.

2. Siegbahn, K. Electron Spectroscopy for Atoms, Molecules, and Condensed Matter. Science 1982, 217, 111-121.

3. Smith, N. V.; Woodruff, D. P. Surface Spectroscopies with Synchrotron Radiation. Science 1982, 216, 367-372.

4. Turner, N.; Schreifels, J. Surface Analysis: X-ray Photoelectron Spectroscopy and Auger Electron Spectroscopy. Anal. Chem. 2000, 72, 99R-110R.

5. Chaudhury, M.; Whitesides, G. Correlation between Surface Free-Energy and Surface Constitution. Science 1992, 255, 1230-1232.

6. Lau, W. M. A Surface Charging Technique in Photoemission Spectroscopic Studies of Dielectric-Semiconductor Structures. J. Appl. Phys. 1990, 67, 1504-1509.

7. Suzer, S. Differential Charging in X-ray Photoelectron Spectroscopy: A Nuisance or a Useful Tool. Anal. Chem. 2003, 75, 7026-7029.

8. Karadas, F.; Ertas, G.; Suzer, S. Differential Charging in $\mathrm{SiO}_{2} /$ Si System as Determined by XPS. J. Phys. Chem. B 2004, 109, 1515-1518.

9. Demirok, U. K.; Ertas, G.; Suzer, S. Time-Resolved XPS Analysis of the $\mathrm{SiO}_{2} / \mathrm{Si}$ System in the Millisecond Range. J. Phys. Chem. B 2004, 108, 5179-5181.
10. Suzer, S.; Sezen, H.; Ertas, G.; Dana, A. XPS Measurements for Probing Dynamics of Charging. J. Electron Spectrosc. Relat. Phenom. 2010, 176, 52-57.

11. Sezen, H.; Ertas, G.; Dana, A.; Suzer, S. Charging-Discharging of Thin PS/PMMA Films as Probed by Dynamic X-ray Photoelectron Spectroscopy. Macromolecules 2007, 40, 4109-4112.

12. Filip-Granit, N.; van der Boom, M. E.; Yerushalmi, R.; Scherz, A.; Cohen, H. Submolecular Potential Profiling Across Organic Monolayers. Nano Lett. 2006, 6, 2848-2851.

13. Cohen, $\mathrm{H}$. Transient Charge Accumulation in a Capacitive Self-Assembled Monolayer. Nano Lett. 2006, 6, 2462-2466.

14. Clabes, J.; Henzler, M. Determination of Surface States on Si(111) by Surface Photovoltage Spectroscopy. Phys. Rev. $B$ 1980, 21, 625-631.

15. Bardeen, J. Surface States and Rectification at a Metal Semiconductor Contact. Phys. Rev. 1947, 71, 717-727.

16. Zeng, T.-W.; Hsu, F.-C.; Tu, Y.-C.; Lin, T.-H.; Su, W.-F. Kelvin Probe Force Microscopy Study on Hybrid P3HT:Titanium Dioxide Nanorod Materials. J. Electron Spectrosc. Relat. Phenom. 2009, 479, 105-108.

17. Schlaf, R.; Hinogami, R.; Fujitani, M.; Yae, S.; Nakato, Y. Fermi level Pinning on HF Etched Silicon Surfaces Investigated by Photoelectron Spectroscopy. J. Vac. Sci. Technol. A 1999, 17, 164-169.

18. Cohen, H.; Sarkar, S. K.; Hodes, G. Chemically Resolved Photovoltage Measurements in CdSe Nanoparticle Films. J. Phys. Chem. B 2006, 110, 25508-25513.

19. Cohen, H. Chemically Resolved Electrical Measurements in Organic Self-Assembled Molecular Layers. J. Electron Spectrosc. Relat. Phenom. 2010, 176, 24-34.

20. Christian, J., Ed. Handbook of Photoelectron Spectroscopy; Physical Electronics: Eden Prairie, MN, 1992.

21. Coluzza, C.; Garozzo, M.; Maletta, G.; Margadonna, D.; Tomaciello, R.; Migliorato, P. NCdS-P-Si Heterojunction Solar-Cells. Appl. Phys. Lett. 1980, 37, 569-572.

22. Garcia, F.; Ortizconte, A.; Saneto, A. CdS/P-Si Solar Cells Made by Serigraphy. Appl. Phys. Lett. 1988, 52, 1261-1263.

23. Kittel, C. Introduction to Solid State Physics, 7th ed.; Wiley: New York, 1976.

24. Suzer, S.; Dana, A. X-ray Photoemission for Probing Charging/Discharging Dynamics. J. Phys. Chem. B 2006, 110, 19112-19115.

25. Dana, A. Lineshapes, Shifts, and Broadenings in Dynamical X-ray Photoelectron Spectroscopy. Appl. Surf. Sci. 2009, 256, 1289-1295.

26. Suzer, S.; Sezen, H.; Dana, A. Two-Dimensional X-ray Photoelectron Spectroscopy for Composite Surface Analysis. Anal. Chem. 2008, 80, 3931-3936.

27. Wildoer, J.; Venema, L.; Rinzler, A.; Smalley, R.; Dekker, C. Electronic Structure of Atomically Resolved Carbon Nanotubes. Nature 1998, 391, 59-62.

28. Chico, L.; Crespi, V.; Benedict, L.; Louie, S.; Cohen, M. Pure Carbon Nanoscale Devices: Nanotube Heterojunctions. Phys. Rev. Lett. 1996, 76, 971-974.

29. Dekker, C. Carbon Nanotubes as Molecular Quantum Wires. Phys. Today 1999, 52, 22-28.

30. Zhou, C.; Kong, J.; Yenilmez, E.; Dai, H. Modulated Chemical Doping of Individual Carbon Nanotubes. Science 2000, 290, 1552-1555.

31. Derycke, V.; Martel, R.; Appenzeller, J.; Avouris, P. Controlling Doping and Carrier Injection in Carbon Nanotube Transistors. Appl. Phys. Lett. 2002, 80, 2773-2775.

32. Tans, S.; Verschueren, A.; Dekker, C. Room-Temperature Transistor Based on a Single Carbon Nanotube. Nature 1998, 393, 49-52.

33. Li, X.; Zhu, H.; Wei, J.; Wang, K.; Xu, E.; Li, Z.; Wu, D. Determination of Band Gaps of Self-Assembled Carbon Nanotube Films Using Tauc/Davis - Mott Model. Appl. Phys. A 2009, 97, 341-344.

34. Kymakis, E.; Amaratunga, G. Single-Wall Carbon Nanotube/ Conjugated Polymer Photovoltaic Devices. Appl. Phys. Lett. 2002, 80, 112-114. 
35. Behnam, A.; Johnson, J.; Choi, Y.; Noriega, L.; Ertosun, M. G.; Wu, Z.; Rinzler, A. G.; Kapur, P.; Saraswat, K. C.; Ural, A. Metal-Semiconductor-Metal Photodetectors Based on Single-Walled Carbon Nanotube Film-GaAs Schottky Contacts. J. Electron Spectrosc. Relat. Phenom. 2008, 103, 114315.

36. Behnam, A.; Johnson, J. L.; Choi, Y.; Ertosun, M. G.; Okyay, A. K.; Kapur, P.; Saraswat, K. C.; Ural, A. Experimental Characterization of Single-Walled Carbon Nanotube FilmSi Schottky Contacts Using Metal-Semiconductor-Metal Structures. Appl. Phys. Lett. 2008, 92, 243116.

37. Behnam, A.; Choi, Y.; Noriega, L.; Wu, Z.; Kravchenko, I.; Rinzler, A. G.; Ural, A. Nanolithographic Patterning of Transparent, Conductive Single-Walled Carbon Nanotube Films by Inductively Coupled Plasma Reactive Ion Etching. J. Vac. Sci. Technol., B 2007, 25, 348-354.

38. Tzolov, M. B.; Kuo, T.-F.; Straus, D. A.; Yin, A.; Xu, J. Carbon Nanotube-Silicon Heterojunction Arrays and Infrared Photocurrent Responses. J. Phys. Chem. C 2007, 111, 58005804.

39. Wei, J.; Jia, Y.; Shu, Q.; Gu, Z.; Wang, K.; Zhuang, D.; Zhang, G.; Wang, Z.; Luo, J.; Cao, A.; et al. Double-Walled Carbon Nanotube Solar Cells. Nano Lett. 2007, 7, 2317-2321.

40. Jia, Y.; Wei, J.; Wang, K.; Cao, A.; Shu, Q.; Gui, X.; Zhu, Y.; Zhuang, D.; Zhang, G.; Ma, B.; et al. Nanotube-Silicon Heterojunction Solar Cells. Adv. Mater. 2008, 20, 4594-4598.

41. Jarillo-Herrero, P.; Sapmaz, S.; Dekker, C.; Kouwenhoven, L.; van der Zant, H. Electron-Hole Symmetry in a Semiconducting Carbon Nanotube Quantum Dot. Nature 2004, 429, 389-392.

42. Ago, H.; Kugler, T.; Cacialli, F.; Salaneck, W.; Shaffer, M.; Windle, A.; Friend, R. Work Functions and Surface Functional Groups of Multiwall Carbon Nanotubes. J. Phys. Chem. B 1999, 103, 8116-8121.

43. Shiraishi, M.; Ata, M. Work Function of Carbon Nanotubes. Carbon 2001, 39, 1913-1917.

44. Sarkar, S. K.; Hodes, G.; Kronik, L.; Cohen, H. DefectDominated Charge Transport in Si-Supported CdSe Nanoparticle Films. J. Phys. Chem. C 2008, 112, 6564-6570.

45. Akca, I. B.; Dana, A.; Aydinli, A.; Turan, R. Comparison of Electron and Hole Charge-Discharge Dynamics in Germanium Nanocrystal Flash Memories. Appl. Phys. Lett. 2008, 92, 052103

46. Brar, B.; Wilk, G.; Seabaugh, A. Direct Extraction of the Electron Tunneling Effective Mass in Ultrathin $\mathrm{SiO}_{2}$. Appl. Phys. Lett. 1996, 69, 2728-2730.

47. Stadele, M.; Sacconi, F.; Di Carlo, A.; Lugli, P. Enhancement of the Effective Tunnel Mass in Ultrathin Silicon Dioxide Layers. J. Appl. Phys. 2003, 93, 2681-2690.

48. Schenk, A.; Heiser, G. Modeling and Simulation of Tunneling Through Ultra-Thin Gate Dielectrics. J. Appl. Phys. 1997, 81, 7900-7908.

49. Nast, O.; Brehme, S.; Pritchard, S.; Aberle, A. G.; Wenham, S. R. Aluminium-Induced Crystallisation of Silicon on Glass for Thin-Film Solar Cells. Sol. Energy Mater. Sol. Cells 2001, 65, 385-392.

50. Feldmann, C.; Metzmacher, C. Polyol Mediated Synthesis of Nanoscale MS Particles (M = Zn, Cd, Hg). J. Mater. Chem. 2001, 11, 2603-2606. 